\title{
On WJCP-Injective Rings
}

Raida D. Mahmood

Raida.1961@uomosul.edu.iq
Shahla M.Khalil

moayadshahla@gmail.com

Department of Mathematics

College of Computer Science and Mathematics

University of Mosul, Mosul, Iraq

\section{Received on: 20/04/2011}

Accepted on: 21/06/2011

\begin{abstract}
As a generalization of right $J C P$ - injective rings, we introduce the nation of right $W J C P$ - injective rings, that is for any right nonsingular element $a$ of $\mathrm{R}$, there exists a positive integer $n$ and $a^{n} \neq 0$ and any right $R$ - homomorphism $f: a^{n} R \rightarrow R$, there exists $m \in R$ such that $f\left(a^{n} c\right)=m a^{n} c$ for all $c \in R$. In this paper, we first introduce and characterize a right $W J C P$ - injective rings . Next, connection between such ring and quasi $\pi$-regular rings and $S$-weakly regular rings.
\end{abstract}

Keywords: right $J C P$ - injective rings, right $W J C P$ - injective rings, quasi $\pi$-regular rings,$S$ - weakly regular rings.

$$
\text { الحلقات الغامرة من النمط WJCP }
$$

شهلة مؤيد خليل

رائدة داؤد محمود

$$
\text { كلية علوم الحاسوب والرياضيات }
$$

تاريخ قبول البحث: 2011/06/21

تاريخ استلام البحث: 2011/04/20

\section{الملخص}

كتعميم للحلقات الغامرة اليمنى من النمط - JCP . سوف نعطي دراسة الحلقات الغامرة اليمنى من النمط

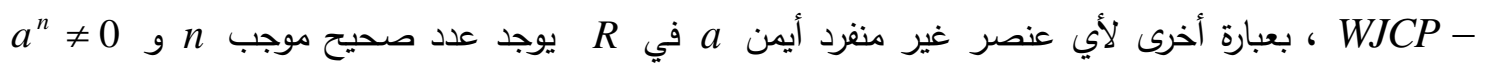

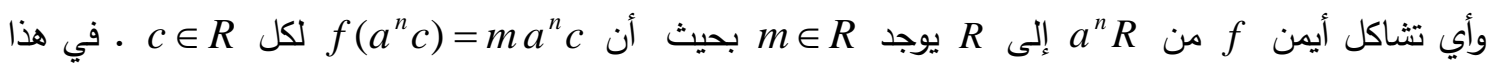
البحث سوف نقدم ونصف أولا دراسة تعميم الحلقات الغامرة اليمنى من النمط - WJCP. وبالتالي علاقة مثل هذه الحلقات مع الحلقات الأخرى مثل حلقات كوازي المنتظمة من النمط - $\pi$ و الحلقات المنتظمة الضعيفة من

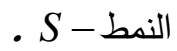

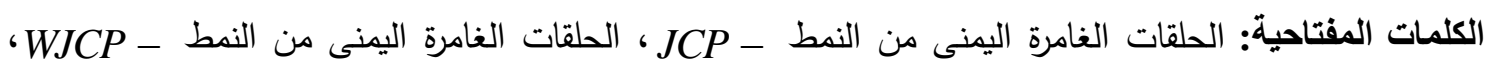

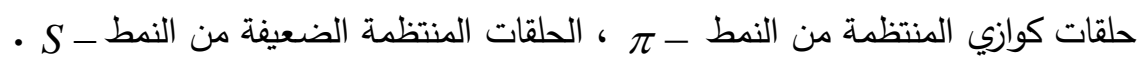

\section{Introduction:}

Throughout this paper $R$ denotes an associative ring with identity, and $R$ modules are unital. For $a \in R, \mathrm{r}(\mathrm{a})$ and $\mathrm{l}(\mathrm{a})$ denote the right annihilator of a and left annihilator of $a$, respectively. We write $\mathrm{J}(\mathrm{R}), \mathrm{Y}(\mathrm{R}), \mathrm{Z}(\mathrm{R})$ for the Jacobson radical , the 
right singular ideal and the left singular ideal, respectively. An element $a \in R$ is called right (left) regular if $\mathrm{r}(\mathrm{a})=0(\mathrm{l}(\mathrm{a})=0)$. [10]

A ring $R$ is reduced if $a^{2}=0$ implies $a=0$ for all $a \in R$, and $R$ is called right $C_{2}$ - ring if every right ideal $\mathrm{T}$ which is isomomorphic to summand of $R$ is a summand [4].

A right $R$-module $M$ is said to be right $Y J$-injective [1], if for any $0 \neq a \in R$ there exists a positive integer $n$ such that $a^{n} \neq 0$ and any right $R$-homomorphism of $a^{n} R$ into $M$ extended to one of $R$ into $M$.

Call a right $R$-module $M, J C P$-injective, if for each $k \notin Y(R)$, any right $R-$ homomorphism $k R \rightarrow M$ extended to $R$. Examples of these module include right $Y J$-injective modules. The concept of $J C P$-injective was first introduced by Wei, [7]. As a generalization of this concept [5], introduced WJCP - injective as a right $R-$ module M , WJCP - injective, if for each $a \notin Y(R)$, then there exists a positive integer $n$ such that $a^{n} \neq 0$ and every right $R$ - homomorphism from $a^{n} R$ into $M$ can be extended to one of $R$ into $M$.

A ring $R$ is called strongly $\pi$-regular ring, if for every $a \in R$ there exists a positive integer $n$ such that $a^{n}=a^{n+1} b$. [2].

A ring $R$ is called $S$-weakly regular ring if for all $a \in R$ then $a \in a R a^{2} R\left(a \in R a^{2} R a\right)$. [3]

\section{2- WJCP-Injective Rings:}

In this section, some basic properties of $W J C P$ - injective rings are given ;

\section{Definition 2.1:[5]}

A right $R$-module $M$ is said to be $W J C P$-injective if for each $a \notin Y(R)$ there exists a positive integer $n$ such that $a^{n} \neq 0$ and every right $R$-homomorphism from $a^{n} R$ into $M$ can be extended to one of $R$ into $M$. If $R_{R}$ is WJCP - injective ring, we call $R$ is right WJCP - injective ring.

Clearly, right $Y J$ - injective rings are right $W J C P$ - injective. The ring in Example $(2.5,[5])$ is a right $W J C P$ - injective which is not right $Y J$-injective.

\section{Theorem 2.2:}

A ring $R$ is a right WJCP - injective if and only if for $a \notin Y(R)$ there exists a positive integer $n$ such that $a^{n} \neq 0$ and $R a^{n}=\operatorname{lr}\left(a^{n}\right)$.

\section{Proof:}

Suppose that a ring $R$ is right WJCP-injective. Then, for every $0 \neq a \notin Y(R)$, there exists a positive integer $n$ such that $a^{n} \neq 0$ and every right $R$-homomorphism from $a^{n} R$ into $R$ can be extended to endomorphism of $R$. It is clear that $R a^{n} \subseteq l\left(r\left(a^{n}\right)\right)$. Let $x \in l\left(r\left(a^{n}\right)\right)$, and $f: a^{n} R \rightarrow R$ are defined by $f\left(a^{n} r\right)=x r$, then, $f$ is well defined right R-homomorphism because $\operatorname{xr}\left(a^{n}\right)=0$ So $r\left(a^{n}\right) \subseteq r(x)$.

Since, $R$ is right WJCP-injective, there exists $c \in R$ such that $f\left(a^{n}\right)=c a^{n}$. Then, $x=f\left(a^{n}\right)=c a^{n} \in R a^{n}$ which implies that $\operatorname{lr}\left(a^{n}\right) \subseteq R a^{n}$. Consequently, $\operatorname{lr}\left(a^{n}\right)=R a^{n}$. 
Conversely, let $a \notin Y(R)$ there exists a positive integer $n$ such that $R a^{n}=\operatorname{lr}\left(a^{n}\right)$. Let $f: a^{n} R \rightarrow R$ be any right $R-$ homomorphism. Then, $r\left(a^{n}\right) \subseteq r\left(f\left(a^{n}\right)\right)$, which implies $f\left(a^{n}\right) \in \operatorname{lr}\left(f\left(a^{n}\right)\right) \subseteq \operatorname{lr}\left(a^{n}\right)=R a^{n}$ and therefore $f\left(a^{n}\right)=d a^{n}$ for some $d \in R$, this

shows that $R$ is right $W J C P$-injective. \#

\section{Example: [7, Example 2.4]}

Let $V$ be a two- dimensional vector space over a field $F$, the trivial extension $R=T(F, V)=F \oplus V$ is commutative, local, artinian ring with $J^{2}=0$ and $J(R)=Y(R)$ . Now, if $x \in R$ with $x \notin Y(R)$, then $x$ is invertible. So, $l\left(r\left(x^{n}\right)\right)=R=R x^{n}$. This implies that $R$ is right $W J C P$-injective. \#

\section{Proposition 2.3:}

Let $R$ be a right $W J C P$-injective and $R a^{n} \subseteq R a$ for all $a \in R$ and a positive integer $n$. Then, any right regular element of $R$ is left invertible.

\section{Proof:}

Let $a \in R$ and there exists a positive integer $n$ such that $r\left(a^{n}\right)=0$. Since, $R$ is right $W J C P$ - injective ring, then $R=\operatorname{lr}\left(a^{n}\right)=R a^{n} \subseteq R a$ by Theorem 2.2. In particular $r a=1$ for some $r \in R$. Hence, $a$ is left invertible. \#

Wei and Chen [5] proved the following theorem:

\section{Theorem 2.4:}

Let $R$ be right WJCP-injective ring. Then, 1- $Y(R) \subseteq J(R)$

2- $R$ is a right $C_{2}-$ ring.

Following [6], a right $R$-module is called $N$ - flat if for each $a \in N(R)$ then, the mapping $I_{M} \otimes i: M \otimes_{R} R a \rightarrow M \otimes_{R} R$ is monic, where $i: R a \rightarrow R$ is the inclusion map .

\section{Lemma 2.5:[6]}

Let $I$ be a right ideal of $R$. Then, $R / I$ is $N$-flat right $R$-module if and only if $I a=I \cap R a$ for all $a \in N(R)$.

\section{Theorem 2.6:}

If $R$ is a right WJCP - injective, $l(a) \subseteq r(a)$ for every $a \in R$ and every simple singular right $R$-module is $N$-flat, then $Z(R)=0$.

\section{Proof:}

If $Z \neq 0$, then $0 \neq b \in Z$ such that $b^{2}=0$. We show that $Z+r(b)=R$. Otherwise there exists a maximal right ideal $M$ such that $Z+r(b) \subseteq M$. If $M$ is not an essential right ideal of $R$, then $M=r(e)$ where $e^{2}=e \in R$. If $b e \neq 0$, then $b e R \cong e R$ as right $R$ module by Theorem 2.4 ( $R$ is $C_{2}$ - ring). $b e R=g R$, where $g^{2}=g \in R$ so, $g \in Z$ because $b e R \subseteq Z$. This is a contradiction. So, $b e=0$. Then $e \in r(b) \subseteq M=r(e)$ which 
is impossible. Thus, $M$ is an essential right ideal of $R$, so $R / M$ is $N$-flat, by Lemma 2.5, $b=a b$ for some $a \in M$, so $1-a \in r(b) \subseteq M$ and then, $1 \in M$, which is a contradiction. Hence, $Z+r(b)=R$, let $1=x+y, x \in Z, y \in r(b)$ then, $b=b x$ and so $b(1-x)=0$ since $x \in Z$ and $l(x) \cap l(1-x)=0, l(1-x)=0$, hence $b=0$ which is a contradiction so $Z(R)=0$. \#

\section{3- The Connection between WJCP - Injective Rings and other Rings.}

In this section, we give the relation between $W J C P$-injective, $S$-weakly regular rings, strongly $\pi$ - regular rings.

Following [7], a ring $R$ is called right quasi regular if $a \in a R a$ for all $a \notin Y(R)$. Now, we give the generalized of quasi regular ring.

\section{Definition 3.1:}

A ring $R$ is called right quasi $\pi$-regular rings if $a^{n} \in a^{n} R a^{n}$ for all $a \notin Y(R)$ and a positive integer $n$. Clearly $R$ is $\pi$-regular ring if and only if $R$ is right non singular and right quasi $\pi-$ regular.

\section{Example:}

Let $Z_{6}$ be a ring of integers modulo 6 , then $Y(R)=\{0\}$ so for all $a \notin Y(R)$, there exists a positive integer $n$ such that $a^{n}=a^{n} R a^{n}$.

\section{Proposition 3.2:}

The following conditions are equivalent for a ring $R$ :

$1-R$ is right quasi $\pi$-regular ring.

2- Every $R$-module is WJCP-injective.

3- Every cyclic $R$-module is WJCP-injective.

\section{Proof:}

$1 \rightarrow 2$ :

Let $M$ be an $R$-module, $a \in R$ with $a \notin Y(R)$ and $f: a^{n} R \rightarrow M$ any right $R$-homomorphism. Since, $R$ is right quasi $\pi$ - regular rings $a^{n}=a^{n} b a^{n}$ for some $b \in R$. Let $a^{n} b=e$ and $f(e)=m$, where $m \in M$. Then, $g: R \rightarrow M$ is defined by $g(r)=m r, r \in R \quad$ is a right $R$-homomorphism, and $g\left(a^{n} r\right)=m a^{n} r=f(e) a^{n} r=f\left(a^{n} b\right) a^{n} r=f\left(a^{n} b a^{n}\right) r=f\left(a^{n}\right) r=f\left(a^{n} r\right)$ which implies that $M$ is WJCP-injective. $2 \rightarrow 3$ :

is trivial.

$3 \rightarrow 1$ :

Let $a \notin Y(R)$. Since, $a^{n} R$ is WJCP - injective, then the identity map $a^{n} R \rightarrow a^{n} R$ can be extended to one of $R$ into $R$. Hence, $a^{n}=a^{n} b a^{n}$ for some $b \in R$. Thus, $R$ is right quasi $\pi$ - regular ring. \#

A ring $R$ right weakly principally small injective [5], if for any $0 \neq a \in J(R)$, there exists a positive integer $n$ such that $a^{n} \neq 0$ and any $R$-homomorphism from $a^{n} R$ to $R_{R}$ can be extended to $R_{R}$ into $R_{R}$. Clearly, every right $Y J$-injective is right weakly principally small injective. 
The following theorem is a generalization of [7,Theorem 2.9] .

\section{Theorem 3.3:}

$R$ is right $Y J$-injective if and only if $R$ is right $W J C P$-injective and right weakly principally small injective.

\section{Proof:}

Assume $R$ is $Y J$-injective, then $R$ is right $W J C P$-injective and weakly principally small injective.

Conversely, let $R$ be a right WJCP-injective, then by Theorem 2.4, $Y(R) \subseteq J(R)$. Let $a \in R$. If $a \notin Y(R)$, then by Theorem 2.2., then $a \notin Y(R)$. If $l\left(r\left(a^{n}\right)\right)=R a^{n}$ . Then, $x \in l\left(r\left(a^{n}\right)\right)$ is clear. Let $R a^{n} \subseteq l\left(r\left(a^{n}\right)\right)$. $R a^{n}=l\left(r\left(a^{n}\right)\right)$ we claim that $a \in J(R)$ is a well defined $f$. Then, $f\left(a^{n} r\right)=x r$ be defined by $f: a^{n} R \rightarrow R$. Let $r\left(a^{n}\right) \subseteq r(x)$ right $R$-homomorphism. Since $R$ is right weakly principally small injective, there exists a right $R$-homomorphism $g: R \rightarrow R$ such that $f\left(a^{n}\right)=g\left(a^{n}\right)$. Hence, $x=f\left(a^{n}\right)=g\left(a^{n}\right)=g(1) a^{n} \in R a^{n}$ and so $l\left(r\left(a^{n}\right)\right) \subseteq R a^{n}$, hence $R a^{n}=l\left(r\left(a^{n}\right)\right)$ therefore, $R$ is $Y J$-injective. \#

\section{Lemma 3.4:[8]}

If $R$ is $S$-weakly regular ring if and only if $R$ is reduced weakly regular ring. Now, we have the following theorem:

\section{Theorem 3.5:}

Let $R$ be a ring whose simple singular right $R$-modules are WJCP-injective. Then, $R$ is reduced if and only if $R$ is $S$ - weakly regular ring.

\section{Proof:}

If $R$ is $S$-weakly regular ring then, $R$ is reduced by Lemma 3.4.

Conversely, assume that $R$ is reduced. For any $0 \neq a \in R$, if $R a^{2} R+r(a) \neq R$, then there exists a maximal right ideal $M$ of $R$ containing $R a^{2} R+r(a)$. If $M$ is not an essential right ideal in $R$, then $M=r(e), e^{2}=e \in R$. Therefore, $e a=0$, since $R$ is abelian, $a e=0$ hence, $e \in r(a) \subseteq M=r(e)$, which is a contradiction. So, $M$ is an essential right ideal in $R$ by hypothesis, $R / M$ is WJCP-injective. Since, $R$ is reduced, $Y(R)=0$. Hence, there exists a positive integer $n$ such that $a^{2 n} \neq 0$ and any right $R$-homomorphism $a^{2 n} R \rightarrow R / M \quad$ can be extended to $R \rightarrow M$. Set $f: a^{2 n} R \rightarrow R / M$ is defined by $f\left(a^{2 n} x\right)=x+M, x \in R$. Then, $f$ is a well defined right $R$-homomorphism. Hence, there exists $g: R \rightarrow R / M$ such that $1+M=f\left(a^{2 n}\right)=g\left(a^{2 n}\right)=g(1) a^{2 n}=c a^{2 n}+M$ where, $\quad g(1)=c+M \quad$, so $1-c a^{2 n} \in M$. Since, $c a^{2 n} \in R a^{2} R \subseteq M, 1 \in M$ which is a contradiction. Hence, $R a^{2} R+r(a)=R$. In particular, $c a^{2} d+x=1$, for some $c, d \in R, x \in r(a)$, then $a=a c a^{2} d$. Therefore, $R$ is $S$ - weakly regular rings. \#

\section{Definition 3.6:[9]}


$R$ is called right $C A M$ - ring, if for any maximal essential right ideal $M$ of $R$ (if it exists ) and for any right sub ideal $I$ of $M$ which is either a complement right sub ideal of $M$ or a right annihilator ideal in $R, I$ is an ideal of $M$.

Show that semi prime right $C A M-$ ring, $R$ is either semi simple artinian or reduced

\section{Lemma 3.7:[9]}

If $R$ is a semi prime right $C A M$ - ring then, $R$ is either semi simple artinian or reduced .

\section{Theorem 3.8:}

Let $R$ be a semi prime right $C A M$ - ring, quasi duo ring whose simple singular right $R$-modules are WJCP-injective. Then $R$ is strongly $\pi$-regular ring .

\section{Proof:}

If $R$ is not a semi simple artinian ring then, $R$ is reduced so $R$ is a right non singular ring. Let $0 \neq a \in R$. If $a^{n} R+r\left(a^{n}\right) \neq R$, then there exists a maximal right ideal $M$ of $R$ such that $a^{n} R+r\left(a^{n}\right) \subseteq M$. If $M$ is not an essential right ideal of $R$, then $M=r(e)$ where $e=e^{2} \in R$ because $R$ is reduced $e a=a e=0$ and $e \in r(a) \subseteq M=r(e)$ is contradiction. Hence, $M$ is an essential right ideal of $R$ and so $R / M$ is a singular simple right $R$-module. By hypothesis $R / M$ is right $W J C P$-injective. Then, there exists $c \in R$ such that $1-c a^{n} \in M$. But, then $1 \in M$ because $R$ is a quasi duo ring and $M$ is an ideal. It is contradiction. Hence, $a^{n} R+r\left(a^{n}\right)=R$ and $R$ is a strongly $\pi-$ regular ring.\#

We conclude the paper with a few characteristic properties of WJCP-injective ring.

\section{Proposition 3.9:}

Let $R$ be a reduced ring and every left principle ideal is a left annihilator of an element in $R$. Then, the followings are

$1-R$ is strongly regular.

$2-R$ is right $Y J$-injective.

3- $R$ is WJCP-injective.

4- $R$ is simple WJCP-injective.

5- $R$ is simple singular WJCP - injective.

6- $R$ is $S$-weakly regular ring.

\section{Proof:}

$1 \rightarrow 2 \rightarrow 3 \rightarrow 4 \rightarrow 5,6 \rightarrow 1$ are trivial

$5 \rightarrow 6$ : by Theorem 3.5 . \# 


\section{REFERENCES}

[1] Ding, N.Q. and Chen, J.L (1994),"Rings Whose Simple Singular Modules are YJinjective", Math. Japo.40, pp.141-195.

[2] Hirano, Y. (1978), "Some Studies on Strongly $\pi$-Regular Rings", Math. J. Okayama Univ. 20, pp. 141-144.

[3] Kandasamy, V., W.B. and Gupta, V. (1993), "S-Weakly Regular Group Rings", Arch, Math. (BRNO) Tomus 29, pp. 39-41.

[4] Nicholson W.K. and Yousif, M.F. (2001),"Weakly Continuous and $\mathrm{C}_{2}$-Rings", comm. In Alg.29:6, pp. 2429-2466.

[5] Wei, J.C. and Chen, J.H. (2007), "Nil-Injective Rings", Int. Electron. J. Algebra,Vol. 2, pp.1-21.

[6] Wei, J.C. and Chen, J.H. (2008),"NPP Rings, Reduced Rings and SNF Rings", Int. Electron. J. of Algebra, Vol. 2, pp.9-26.

[7] Wei, J. (2009), "JCP-Injective Rings", International Electronic of Algebra Vol. 6, pp.1-22.

[8] Younis, A.M. (2003), "On S-Weakly Regular Ring", M.Sc. Thesis, Mosul University.

[9] Yue Chi Ming, R. (1983), "On Quasi-Frobeniusean and Artinian Rings", Publications Delinstitut Math ematique, 33(47), pp. 239-245.

[10] Yue Chi Ming, R. (1976), "On Annihilator Ideals", Math. J. Okayama Univ., 19, pp. 51-53. 